\title{
Electro-thermally tunable reflective colors in a self-organized cholesteric helical superstructure
}

\author{
Po-Chang $\mathrm{Wu}^{1}$, Guan-Wei Wu${ }^{2}$, Ivan V. Timofeev ${ }^{3}, 4$, Victor Ya. Zyryanov ${ }^{3}$, and Wei Lee ${ }^{1, *}$ \\ ${ }^{1}$ Institute of Imaging and Biomedical Photonics, College of Photonics, National Chiao Tung University, Guiren Dist., Tainan \\ 71150, Taiwan \\ ${ }^{2}$ Institute of Lighting and Energy Photonics, College of Photonics, National Chiao Tung University, Guiren Dist., Tainan \\ 71150, Taiwan \\ ${ }^{3}$ Kirensky Institute of Physics, Federal Research Center "Krasnoyarsk Scientific Center", Siberian Branch of the Russian \\ Academy of Sciences, Krasnoyarsk 660036, Russia \\ ${ }^{4}$ Laboratory for Nonlinear Optics and Spectroscopy, Siberian Federal University, Krasnoyarsk 660041, Russia \\ ${ }^{*}$ Corresponding author: wlee@nctu.edu.tw
}

Received Month X, XXXX; revised Month X, XXXX; accepted Month X, XXXX; posted Month X, XXXX (Doc. ID XXXXX); published Month X, XXXX

\begin{abstract}
We propose to dynamically control the reflective color of a cholesteric liquid crystal (CLC) by electrically tuning the center wavelength $\left(\lambda_{c}\right)$ of the bandgap. The CLC, sandwiched in a planar-aligned cell with indium-tin-oxide electrodes, possesses negative dielectric anisotropy and thermo-responsive spectral properties. The helix in the Grandjean planar state subject to a vertically applied voltage should be undisturbed in that the long molecular axis is initially perpendicular to the direction of the electric field. Surprisingly, when the frequency of the applied voltage is higher than a critical value, $\lambda_{\mathrm{c}}$ of the CLC cell varies as a function of the voltage. The underlying mechanism is the voltage-induced temperature change through dielectric heating in the frequency regime of pseudo-dielectric relaxation, attributable to the significant equivalent resistance-capacitance circuit of the cell due to the use of electrode layers with finite conductance. The driving voltage enabling the tuning of $\lambda_{\mathrm{c}}$ in the entire visible spectrum is as low as $12 \mathrm{~V}_{\mathrm{rms}}$ in a 5- $\mu \mathrm{m}$-thick cell at a frequency of $2 \mathrm{MHz}$. The proposed CLC cell exhibiting a broad electrically tunable spectral range from near infrared to ultraviolet holds great promise for developing tunable photonic devices such as multicolor reflectors, filters, and sensors.

(C) 2018 Chinese Laser Press

OCIS codes: (160.3710) Liquid crystals; (160.1585) Chiral media; (230.2090) Electro-optical devices; (230.3720)

Liquid-crystal devices
\end{abstract}

\section{INTRODUCTION}

Cholesteric liquid crystals (CLCs) organized in self-assembled, periodically helical superstructures are known as a class of onedimensional chiral photonic crystal. In the Grandjean planar (P) state a CLC is characterized by the so-called Bragg bandgap in a specific wavelength region, enabling the reflection of selective circularly polarized light with the same handedness as that of the CLC helix. This unique feature permits CLCs to be promising for the development of a variety of photonic devices such as reflective displays, reflectors, lasers, and sensors [1-4]. More attractively, dynamic control of CLC bandgap properties is attainable, permitting CLC photonic devices with variable optical properties. The center wavelength $\left(\lambda_{c}\right)$ of the CLC bandgap is determined by the helical pitch $(p)$ as well as the ordinary $\left(n_{0}\right)$ and extraordinary $\left(n_{\mathrm{e}}\right)$ refractive indices of the nematic host, according to the relation $\lambda_{\mathrm{c}}=p \cdot\left(n_{\mathrm{e}}+n_{\mathrm{o}}\right) / 2$ [5]. As such, a good number of studies of tunability of $\lambda_{c}$ or tunable reflective color in CLCs have been proposed via the control of helical pitch by external stimuli. For instance, CLCs with thermo-responsive bandgap properties have been obtained on the basis of the temperature dependency of LC phase transition [6], the helical twisting power (HTP) of the chiral dopant [7], and the mixture solubility [8]. Employment of photoresponsive chiral switches as dopants in CLCs has also been suggested for realizing variable and rewritable $\lambda_{\mathrm{c}}$ by light irradiations thanks to the tunable HTP via the process of photoinduced trans-cis isomerizations [9]. On the other hand, electrical tuning of $\lambda_{\mathrm{c}}$ would be preferable for practical applications, because of the ease of operation and high compatibility of the CLC cell with modern optoelectronic devices. Considering a CLC cell subject to a vertical electric field, most of recent electrical-tuning approaches have been developed using negative nematic LCs - namely, nematic LCs with negative dielectric anisotropy $(\Delta \varepsilon<0)$ - as the host material to prevent helical distortion by the applied voltage. With a direct-current (DC) voltage applied to a CLC consisting of chiral additives in negative LC, wavelength tuning has been asserted and examined in terms of the voltage-induced electrohydrodynamic instability [10], mechanical cell-gap bending [11], and defect annealing [12]. Alternatively, Choi et al. proposed to further exploit ferroelectric LC as electrically commanded aligning surfaces [13] or as dopants [14] in a negative CLC cell to achieve bandgap tuning by alternating-current (AC) voltages. However, the tunable wavelength ranges of the bandgap are quite limited and the voltages required are generally too high $(>100 \mathrm{~V}$ by either $\mathrm{DC}$ or AC voltage) by the above-mentioned electricaltuning approaches, thus hindering their practical uses. In a special case, Xiang et al. demonstrated electrically tunable selective reflection from ultraviolet (UV) to visible and infrared (IR) in an oblique helicoid state rather than the typical $\mathrm{P}$ state by doping proper amounts of the bent-shape molecules $\mathrm{CB} 7 \mathrm{CB}$ and CB6OCB into a CLC [15]. 
Different from all aforementioned studies, in this work we propose a state-of-the-art approach based on the electro-thermal effect, allowing the tunability of the reflective color in a wide wavelength range, covering the entire visible spectrum, by $\mathrm{AC}$ voltage applied across a negative CLC that is confined in a planaraligned cell with indium-tin-oxide (ITO) electrodes. Here the CLC is designed to intrinsically possess thermo-responsive bandgap properties. When the frequency of applied voltage goes beyond a critical value, our results indicated that $\lambda_{\mathrm{c}}$ of the reflection band can be electrically tuned by varying either the amplitude or the frequency of the $\mathrm{AC}$ voltage. The results are complemented by both dielectric spectroscopy and IR thermography, affirming the increase in cell temperature through the induction of dielectric heating by the applied voltage. In general, dielectric heating in a dielectric medium stems from absorption of electromagnetic energy through rotation of molecular dipoles and its efficacy becomes remarkable in the frequency regime of dielectric relaxation. Because the dielectric relaxation from molecular dipole reorientation of a typical $\mathrm{LC}$ does not occur in the $\mathrm{kHz}-\mathrm{to}^{-} \mathrm{MHz}$ frequency regime, the dielectric heating effect in sandwich-type LC cells to date has only been discussed in dual-frequency LC whose relaxation frequency (viz., the crossover frequency) is about tens of $\mathrm{kHz}$ [16-18]. In our case, where a common negative CLC is employed, the dielectric heating is primarily governed by the pseudo-relaxation from the ITO effect due to the generation of losses from the cell comprising electrodes with non-zero resistance. As a result, the $\lambda_{c}$ of the proposed negative CLC with superior electro-thermal properties can vary from $780 \mathrm{~nm}$ at $0 \mathrm{~V}_{\mathrm{rms}}$ to 380 $\mathrm{nm}$ at merely $12 \mathrm{~V}_{\mathrm{rms}}$ at $2 \mathrm{MHz}$, manifesting a wider tunable wavelength range entailing much lower driving voltage in comparison with those by most of current electrical tuning methods.

\section{EXPERIMENT}

The CLC used was prepared by incorporating $45 \mathrm{wt} \%$ of the lefthanded chiral additive S-811 (Merck) into the negative nematic host MLC-6608 (Merck) to induce the SmA-CLC phase transition so as to display thermal-responsive spectral properties in the CLC phase. MLC- 6608 has birefringence $\Delta n=0.083$ (measured at the wavelength of $589 \mathrm{~nm}$ and temperature of $20{ }^{\circ} \mathrm{C}$ ) and dielectric anisotropy $\Delta \varepsilon=-4.2$ (at the frequency $f$ of $1 \mathrm{kHz}$ and temperature of $20{ }^{\circ} \mathrm{C}$ ). The phase sequence of the mixture is $\mathrm{SmA}-21$ ${ }^{\circ} \mathrm{C}-\mathrm{CLC}-39{ }^{\circ} \mathrm{C}-$ Isotropic, as determined by optical textures using a polarizing optical microscope (Olympus BX51) at various temperatures in a heating process. The CLC was heated to the isotropic phase and stirred for $2 \mathrm{~h}$ to ensure homogeneous blending, followed by its injection into an empty cell by capillary action. The empty cell is composed of two glass substrates; each substrate bearing an ITO electrode was spin-coated with an aligning layer (SE-2170) to promote planar alignment of LC molecules and, thus, the initial P state in the CLC phase. The cell gap and the effective electrode area of the cell are $d=5 \pm 0.5 \mu \mathrm{m}$ and $A=1 \mathrm{~cm}^{2}$, respectively. In general, a LC cell is made by sandwiching a LC between two ITO-covered substrates. When the resistivity of the ITO is not negligible the equivalent circuit of such a sandwich-type cell geometry can be represented by a resistance arising from the electrode layers and a capacitance contributed by the LC layer in series. As such, it has been found that the cell applied with voltages at specific frequencies will generate a certain amount of dielectric losses, leading to the induction of a pseudo-dielectric relaxation in the complex dielectric spectra [19]. The corresponding relaxation frequency $\left(f_{\mathrm{PR}}\right)$ can be defined as:

$$
f_{\mathrm{PR}}=1 /\left(2 \pi R_{\mathrm{ITO}} C_{\text {cell }}\right)
$$

where $R_{\mathrm{ITO}} \sim 310 \Omega$ is the resistance of the ITO and $C_{\text {cell }}=\varepsilon_{0} \mathcal{E}_{\mathrm{S}}(A / d)$ is the capacitance of the cell. Because the vacuum permittivity $\varepsilon_{0}$ $=8.854 \times 10^{-12} \mathrm{~F} \cdot \mathrm{m}^{-1}, A=1 \mathrm{~cm}^{2}$ and $d=5 \mu \mathrm{m}$ are all constants, the values of $C_{\text {cell }}$ as well as $f_{\mathrm{PR}}$ are primarily dominated by the dielectric constant of the material $\left(\varepsilon_{\mathrm{s}}\right)$ filled in the cell. In the case of an empty cell with an air gap $\left(\mathcal{E}_{\mathrm{S}}=1\right), \mathrm{C}_{\text {cell }}$ measured is $\sim 0.18 \mathrm{nF}$ and ffR as calculated by Eq. (1) is $\sim 2.85 \mathrm{MHz}$. Note that this unique pseudo-dielectric relaxation is the key responsible for the dielectric heating by an external voltage in this study.

All measurements were carried out at a given surrounding temperature $\left(T_{\mathrm{s}}=22^{\circ} \mathrm{C}\right.$ or $23^{\circ} \mathrm{C}$ as designated $)$ without using a hot stage unless specified. Spectral properties of the cell in the wavelength regime between $380 \mathrm{~nm}$ and $800 \mathrm{~nm}$ were monitored by transmission spectroscopy using a fiber-optic spectrometer (Ocean Optics HR2000+) in conjunction with a halogen light source (Ocean Optics HL2000). No polarizer was employed in the spectral measurement. To investigate the dielectric heating effect and electro-thermal properties of the CLC cell, dielectric spectra and the change in cell temperature $(\Delta T)$ as a function of the applied voltage ( $V$ ) were respectively acquired with a highprecision LCR meter (Agilent E4980A) and a non-contact IR thermometer (precisely, IR camera FLIR ThermaCam ${ }^{\circledR}$ P25). External voltages in the sinusoidal waveform with controllable amplitudes (from $0.05 \mathrm{~V}_{\text {rms }}$ to $15 \mathrm{~V}_{\text {rms }}$ ) and frequencies (from $20 \mathrm{~Hz}$ to $2 \mathrm{MHz}$ ) were specifically supplied by the same LCR meter to prevent from errors under various voltage conditions caused by different instruments.

\section{RESULTS AND DISCUSSION}

Figure 1 illustrates the temperature-dependent bandgap properties and optical textures at four given temperatures of the CLC cell without voltage applied. Here, the cell was placed on a hot stage (Linkam-LTS120E) to precisely control the cell temperature. The experimental data, as shown in Fig. 1(a), indicate that the reflection band blue-shifted gradually with rising temperature $(T)$, varying from $\lambda_{\mathrm{c}}=760$ nm at $T=23{ }^{\circ} \mathrm{C}$ to $\lambda_{\mathrm{c}}=380 \mathrm{~nm}$ at $T=32{ }^{\circ} \mathrm{C}$. This led to the change in color of the reflective optical texture at various temperatures, appearing red $\left(\lambda_{\mathrm{c}}=760 \mathrm{~nm}\right)$ at $23{ }^{\circ} \mathrm{C}$, orange $\left(\lambda_{\mathrm{c}}=610 \mathrm{~nm}\right)$ at $23.8^{\circ} \mathrm{C}$, green $\left(\lambda_{\mathrm{c}}=512 \mathrm{~nm}\right)$ at $25^{\circ} \mathrm{C}$, and navy $\left(\lambda_{\mathrm{c}}=397 \mathrm{~nm}\right)$ at $29.4{ }^{\circ} \mathrm{C}$ (see Fig. $\left.1(\mathrm{~b})\right)$. When using the chiral material S-811 as the dopant to form a CLC with a SmA-CLC phase transition, similar results to Fig. 1(a) have also been obtained in CLCs with distinct nematic hosts [12, 20] and this phenomenon has been interpreted by the temperature dependence of the twist elastic constant [6]. Such temperature-dependent spectral behavior can be fitted by Keating's theory, expressed as [21]:

$$
\lambda_{c}(T)=\gamma \frac{T_{0}}{T}\left(1+\frac{\beta}{T-T_{0}}\right)^{2},
$$

where $\gamma$ and $\beta$ are fitting parameters, $T$ is in Kelvin (K), and $T_{0}<T$ is the SmA-CLC phase transition temperature. We simulated temperature-dependent $\lambda_{c}$ of our cell in the CLC phase temperature range between $22^{\circ} \mathrm{C}$ and $39{ }^{\circ} \mathrm{C}$ by fitting 
the experimental data (open circles) given in Fig. 1(a) and, with the best-fit parameters of $\gamma=321.58 \mathrm{~nm}$ and $\beta=1.08$ $\mathrm{K}$, the simulated curve (solid line) suggests that the proposed CLC cell can possess a widely tunable range of $\lambda_{\mathrm{c}}$ by suitable temperature control, from $1392 \mathrm{~nm}$ (IR) at $22^{\circ} \mathrm{C}$ to $340 \mathrm{~nm}$ (UV) at $39^{\circ} \mathrm{C}$.

To discuss the correlation between the "pseudo-dielectric relaxation" and the voltage-induced dielectric heating, Fig. 2(a) firstly displays the complex dielectric spectra of the pure LC (MLC-6608) and the CLC (MLC-6608 + 45-wt\% S811) cells representatively at $T=23{ }^{\circ} \mathrm{C}$. Because the orientation of the LC molecules having $\Delta \mathcal{E}<0$ remains unchanged in a vertical electric field, the profile of the dielectric spectrum is independent of the amplitude of the probe voltage. When the resistivity of the ITO material is

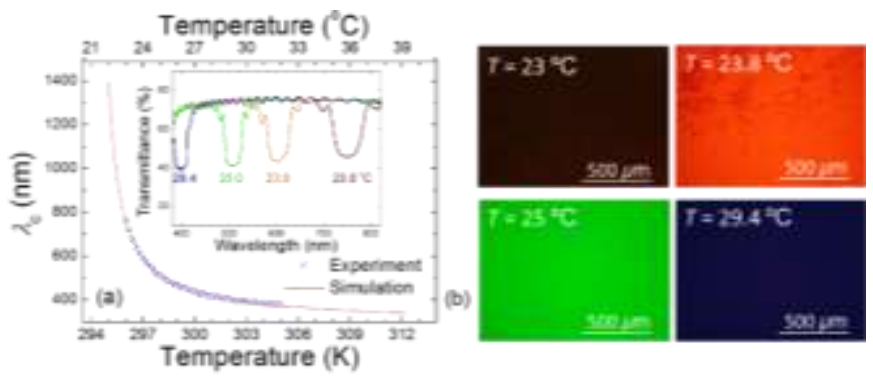

FIG. 1. Temperature-dependent (a) $\lambda_{c}$ of the experimental (open circles) and simulated (solid line) results and (b) optical textures of the cell in the CLC phase observed in the reflection mode at four distinct temperatures. Inset in (a) is the transmission spectra at various temperatures.
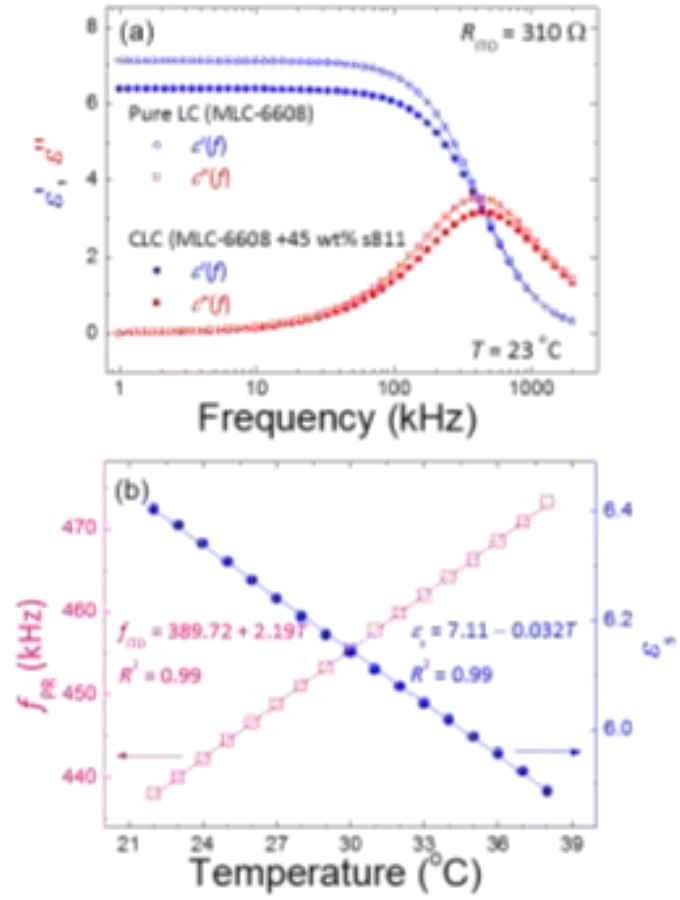

FIG. 2. (a) Experimental and simulated complex dielectric spectra of the pure LC (MLC-6608) and the CLC (MLC-6608 $+45-w t \% \mathrm{~S}-811$ ) cells at $T=23{ }^{\circ} \mathrm{C}$ and (b) temperature dependence of deduced static dielectric constant $\mathcal{E}_{\mathrm{S}}$ and relaxation frequency $f_{\mathrm{PR}}$ of the pseudo-dielectric relaxation of the LC cell in the CLC phase. nontrivial, which is truly the case, a resistance-capacitance circuit is produced in a sandwich-type cell, resulting in energy losses and, in turn, a pseudo-dielectric relaxation [19]. Now that the polarization contributed by space charge is excluded at high frequencies $(>\mathrm{kHz})$ and the orientational polarization relaxation of a negative LC typically occurs beyond $\mathrm{MHz}$ [18], the single Debye-type relaxation detected in the complex dielectric spectra of either cell is undoubtedly attributable to the use of the ITO cell. Referring to the Debye model, the real- $\left(\varepsilon^{\prime}\right)$ and imaginary-part ( $\left.\mathcal{\varepsilon}^{\prime \prime}\right)$ dielectric functions of this relaxation can be described by:

$\varepsilon^{\prime}=\varepsilon_{\infty}+\frac{\left(\varepsilon_{\mathrm{s}}-\varepsilon_{\infty}\right)}{1+\omega^{2} \tau_{\mathrm{PR}}^{2}}$

and

$\varepsilon^{\prime \prime}=\frac{\left(\varepsilon_{\mathrm{s}}-\varepsilon_{\infty}\right) \omega \tau_{\mathrm{PR}}}{1+\omega^{2} \tau_{\mathrm{PR}}^{2}}$,

respectively, where $\omega=2 \pi f$ is the angular frequency, $\tau \mathrm{PR}$ is the relaxation time of this pseudo-dielectric behavior inversely related to the relaxation frequency $f_{\mathrm{PR}}$ in accordance with $2 \pi f_{\mathrm{PR}} \tau_{\mathrm{PR}}=1$, and $\varepsilon_{\mathrm{S}}$ and $\varepsilon_{\infty}$ are dielectric permittivities at the low $^{-}$and high-frequency limits, respectively. By fitting the experimental data of Fig. 2(a) to either Eq. (3) or Eq. (4), $\mathcal{E}_{\mathrm{s}}$ and fpr of the CLC cell at $T=23$ ${ }^{\circ} \mathrm{C}$ are deduced to be $\sim 6.4$ and $\sim 440 \mathrm{kHz}$, respectively. In accordance with Eq. (1), $\mathcal{E}_{\mathrm{s}}$ of the cell is changed from 1 to 6.4 after the injection of CLC so that $f_{\mathrm{PR}}$ can be calculated by $f_{\mathrm{PR}}=2.85 / 6.4 \sim 445 \mathrm{kHz}$, which is in good agreement with that of the fitted result (i.e., $f_{\mathrm{PR}}=440 \mathrm{kHz}$ ). It can also be found that the profile of pseudo-dielectric relaxation of the CLC cell is nearly identical to that of the pure LC counterpart, indicating that adding S-811 into MLC-6608 is insufficient to modify the relaxation frequency $\left(\right.$ from $f_{\mathrm{PR}}=$ $417 \mathrm{kHz}$ in the pure LC to $f_{\mathrm{PR}}=440 \mathrm{kHz}$ in the CLC) due to the limited variation in $\varepsilon_{\mathrm{s}}$. This also implies that the dielectric relaxation from LC molecules cannot be shifted to the investigated frequency regime even when doping $45 \mathrm{wt} \%$ of S-811 into the LC. Figure 2(b) unambiguously reveals the temperature dependence of $f_{\mathrm{PR}}$ of the CLC cell. One can see that $f_{\mathrm{PR}}$ varies linearly with $T$ and it shows a $35 \mathrm{-kHz}$ variation only, from $f_{\mathrm{PR}}=438 \mathrm{kHz}$ (at $T=22^{\circ} \mathrm{C}$ ) to $f_{\mathrm{PR}}=473$ $\mathrm{kHz}$ (at $T=38^{\circ} \mathrm{C}$ ). This temperature-dependent relaxation behavior is in agreement, to the first order of the binomial expansion, with the decrease in $\mathcal{E}^{\prime}$ with increasing $T$ (from 6.4 at $T=22^{\circ} \mathrm{C}$ to 5.9 at $T=38^{\circ} \mathrm{C}$ ). It should be noted here that the change in $f_{\mathrm{PR}}$ as a function of the temperature also follows the Arrhenius equation with the activation energy of $0.038 \mathrm{eV}$.

Figure 3(a) depicts the $f$ dependence of the steady-state cell temperature at $V=15 \mathrm{~V}_{\text {rms }}$ measured by an IR camera. Here, each IR image was taken after the application of a given voltage to the cell for $8 \mathrm{~min}$ to ensure that the cell temperature became saturated or time-independent. For this measurement, $T_{\mathrm{s}}$ was controlled at $23{ }^{\circ} \mathrm{C}$ and the temperature increase $\Delta T$ is defined as $T-T_{\mathrm{s}}>0$. The control of cell temperature by applied voltage was directly confirmed by IR thermography, revealing image color changes from blue in the field-off state or at $f=1 \mathrm{kHz}(T=$ $\left.23^{\circ} \mathrm{C}\right)$ to green at $f=600 \mathrm{kHz}\left(T=32{ }^{\circ} \mathrm{C}\right)$ and to yellow at $f$ $=2 \mathrm{MHz}\left(T=37.7^{\circ} \mathrm{C}\right)$, as shown in the insets of Fig. 3(a). 
Figure 3(a) indicates that there exists a critical frequency $\left(f_{0}\right)$ that triggers the onset of temperature increase with increasing frequency. In the case of $V=15 \mathrm{~V}_{\mathrm{rms}}, \Delta T$ starts to increase with rising frequency beyond $24.5 \mathrm{kHz}$, reaching $\Delta T \sim 14.7{ }^{\circ} \mathrm{C}$ (i.e., $T=37.7{ }^{\circ} \mathrm{C}$ ) at $f=2 \mathrm{MHz}$. In considering the relation between the heat generation and the dielectric properties of a LC cell, we attempt to elucidate the result of Fig. 3(a) in terms of the temperature elevation from the dielectric heating effect by the following time-varying equation [16]:

$\Delta T=\left(\frac{V^{2} A \varepsilon_{0}\left(\varepsilon_{\mathrm{s}}-\varepsilon_{\infty}\right)}{d(C+h t)}\right)\left(\frac{\tau_{\mathrm{R}} \omega^{2} t}{1+\omega^{2} \tau_{\mathrm{R}}^{2}}\right)$,

where $t$ is the dielectric heating time, $\varepsilon_{0}$ is the dielectric constant in vacuum and $C$ and $h$ are the average heat capacity and the specific heat conductivity of the cell, respectively. In contrast to $\tau_{\mathrm{PR}}\left(f_{\mathrm{PR}}\right), \tau_{\mathrm{R}}\left(f_{\mathrm{R}}\right)$ is the relaxation time (frequency) of the principal dielectric relaxation that dominates the voltage-induced dielectric heating. To date, Eq. (5) has only been exploited for explaining the dielectric heating behaviors of a LC cell from the dielectric relaxation of LC molecules [16, 17]. Nevertheless, because the origin of Eq. (5) stems from the power dissipated in a LC cell due to dielectric losses, it is believed that this equation is still valid and can be in use to characterize the increase in temperature in our designated cell by dielectric heating from the pseudo-dielectric relaxation displayed in Fig. 2(a).
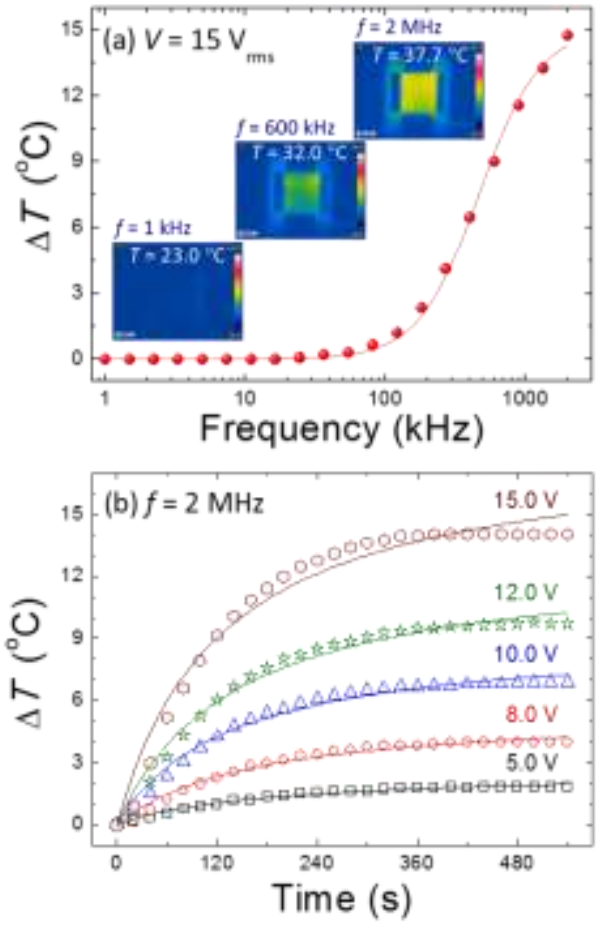

FIG. 3. (a) Temperature increase as a function of the frequency of an applied AC voltage of $15 \mathrm{~V}_{\mathrm{rms}}$ and (b) dynamic temperature increases of the CLC cell under the application of various voltages at a fixed frequency of $2 \mathrm{MHz}$. Note that the solid line in (a) is a simulated curve based on Eq. (6) and that the open symbols and solid lines in (b) represent experimental and curve-fitting results according to Eq. (5), respectively.
By setting $\mathrm{t} \rightarrow \infty$, Eq. (5) in the stationary state can be simplified as [22]:

$\Delta T=\left(\frac{V^{2} A \varepsilon_{0}\left(\varepsilon_{\mathrm{s}}-\varepsilon_{\infty}\right)}{d h}\right)\left(\frac{\tau_{\mathrm{R}} \omega^{2}}{1+\omega^{2} \tau_{\mathrm{R}}^{2}}\right)$.

While fitting the experimental data (represented by spherical symbols) from Fig. 3(a) with Eq. (6), one can find that the fitting curve of $\Delta T(f)$ (solid line in Fig. 3(a)), with the parameters $h \sim 0.055 \mathrm{~W} \cdot \mathrm{K}^{-1}$ and $\tau_{\mathrm{R}} \sim 3.4 \times 10^{-7} \mathrm{~s}$, is in line with the measured data (yielding the corresponding coefficient of determination $R^{2}=0.99$ ). Notably, the $f_{\mathrm{R}}$ value of $\sim 467 \mathrm{kHz}$ calculated from $\tau_{\mathrm{R}}$ is very close to $f_{\mathrm{PR}}=467 \mathrm{kHz}$ at $T=35.3^{\circ} \mathrm{C}$ or $f_{\mathrm{PR}}=472.5 \mathrm{kHz}$ at $T=37.7^{\circ} \mathrm{C}$ (see Fig. $2(\mathrm{~b})$ ). Moreover, Fig. 3(b) delineates time-evolved temperature changes of the cell driven by various voltages at $f=2 \mathrm{MHz}$. Again, these observations were performed at $T_{\mathrm{s}}=23{ }^{\circ} \mathrm{C}$. For a given voltage, the temperature increased monotonically with increasing dielectric heating time, reaching a saturated value and becoming saturated at $t>360 \mathrm{~s}$. These results can be properly fitted to Eq. (5) with $R^{2} \geq 0.97$. For each fitting curve in Fig. 3(b), $C$ and $h$ are adjustable parameters. Because the relation of $f_{R}=f_{\mathrm{P}}$ is verified based on fitting results of Fig. 3(a), $\tau_{\mathrm{R}}$ here is considered as a fixed value and is given from $\tau_{\mathrm{R}}=\left(2 \pi \mathrm{f}_{\mathrm{R}}\right)^{-1}$ at its saturated temperature, extracted from Fig. 2(b) (e. g., f $f_{\mathrm{R}}=470.8 \mathrm{kHz}$ at $T=37.0^{\circ} \mathrm{C}$ for $V=15 \mathrm{~V}_{\mathrm{rms}}$ or $f_{\mathrm{PR}}=461.3 \mathrm{kHz}$ at $T=32.7$ ${ }^{\circ} \mathrm{C}$ for $V=12 \mathrm{~V}$ rms $)$. The fitting results indicate that $C$ is nearly constant at various voltage conditions, yielding $C=$ $5.6 \pm 0.4 \mathrm{~J} \cdot \mathrm{K}^{-1}$. This is understandable because $C$ is primarily dictated by the thermal properties of the ITOsubstrates and the LC layer which are virtually unaffected by the external voltage. The findings unraveled in Fig. 3 suggest that the heat generation in the designated CLC cell is ascribed to the generation of dielectric losses from the equivalent $R_{\text {ITO }}-C_{\text {cell }}$ circuit through the dielectric heating effect. Although a rigorous mechanism behind the dielectric heating behavior from the cell-geometry-dominated dielectric relaxation is still underway in this laboratory, the experimental results reported in the present work can theoretically be formulated by Eq. (5), giving a general approximation describing the temperature increase arising from the dielectric heating.

The pseudo-dielectric relaxation from the ITO effect has long been considered as a nuisance for dielectric investigations of a LC in that signals of certain relaxations from the LC material could be concealed at high frequencies [23]. Because the pseudo-dielectric relaxation can hardly be avoided in a sandwich-type cell, the direct way to suppress or shift this undesired dielectric signal beyond the investigated frequency regime is to use metal substances instead of ITO to lower the resistivity of the electrodes (e.g. silver electrode used in Ref. 16) and/or to increase the cell gap (e.g. $d=40 \mu \mathrm{m}$ used in Ref. 17), according to Eq. (1). Some other methods based on mathematical calculations have also been proposed to eliminate the contribution of the cell-geometry-induced pseudo signal to the complex dielectric spectra so as to deduce the real dielectric behaviors of $\operatorname{LCs}[19,23]$. Since the dielectric heating behavior in a LC cell has only been focused on the dielectric relaxation from dual-frequency LC molecules, the present work would be the first of its kind confirming the contribution of the LC container to dielectric heating. 


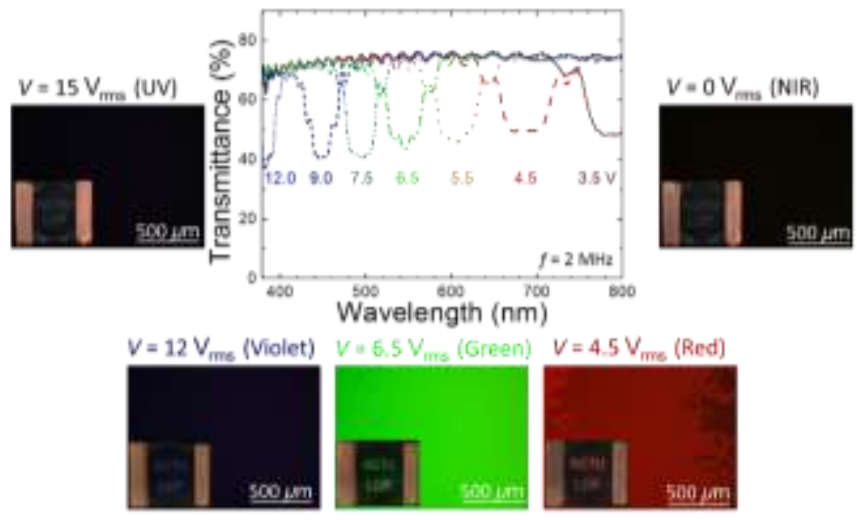

FIG. 4. Transmission spectra, texture images of the CLC and photographs of the real cell driven by various voltages at $2 \mathrm{MHz}$.

Utilizing this unique feature together with the thermalresponsive bandgap properties of a CLC, one can electrically tune the reflection band of the negative CLC through the control of cell temperature by an externally applied voltage. Figure 4 displays transmission spectra and optical textures together with the corresponding photographs of the cell submitted to various $\mathrm{AC}$ voltages at $f=2 \mathrm{MHz}$. Taken without any polarizer, these photographs shown as the insets are mirror images of a piece of black paper showing white inverted letters ("NCTU LCP") reflected from the CLC cell. To illustrate the wavelength tuning ability, we referred to Fig. 1(a) and masterly controlled $T_{\mathrm{s}}=22{ }^{\circ} \mathrm{C}$ so as to allow the reflection band to lie in the near IR region in the fieldoff state $\left(\lambda_{\mathrm{c}}=1392 \mathrm{~nm}\right.$ at $\left.V=0 \mathrm{~V}_{\mathrm{rms}}\right)$. When the voltage increased to $3.5 \mathrm{~V}_{\mathrm{rms}}$, the bandgap appeared in the visible light regime $\left(\lambda_{\mathrm{c}} \sim 800 \mathrm{~nm}\right.$ at $\left.V=3.5 \mathrm{~V}_{\mathrm{rms}}\right)$ and blue-shifted further with increasing voltage, reaching $\lambda_{\mathrm{c}} \sim 380 \mathrm{~nm}$ at $V$ $=12 \mathrm{~V}_{\text {rms. }}$. The color change of the reflective optical texture and of the cell appearance from red at $V=4.5 \mathrm{~V}_{\text {rms }}$ to green at $V=6.5 \mathrm{~V}$ rms, blue at $V=9 \mathrm{~V}_{\mathrm{rms}}$ and to violet at $V=12 \mathrm{~V}_{\mathrm{rms}}$ are consistent with the corresponding bandgap properties. Further increasing the voltage to $15 \mathrm{~V}_{\text {rms }}$ resulted in the disappearance of the bandgap from the visible spectrum.

The bandgap shift to the UV region at $V=15 \mathrm{~V}_{\text {rms }}$ brought about the dark appearance of the CLC phase. The results as shown in Fig. 4 imply that the proposed electrical tuning method enables a broadband tunable wavelength range, spanning from the IR, visible, and UV light regions, for reflective color by simply regulating the applied voltage between 0 and $15 \mathrm{~V}_{\text {rms }}$ at $f=2 \mathrm{MHz}$.

Alternatively, Fig. 5(a) depicts the frequency dependence of tunable $\lambda_{c}$ of the cell under the application of various external voltages. In considering the limit of detection of the used spectrometer (i.e., $380 \mathrm{~nm}-820 \mathrm{~nm}$ ), $T_{\mathrm{s}}$ here was set at $\sim 23{ }^{\circ} \mathrm{C}$ so that $\lambda_{\mathrm{c}} \sim 760 \mathrm{~nm}$ at $V=0 \mathrm{~V}_{\text {rms. }}$ It is clear that $\lambda_{\mathrm{c}}$ remained unchanged and then decreased with ascending frequency beyond a critical value $\left(f_{0}\right)$, dropping with increasing $V$ from $f_{0} \sim 118 \mathrm{kHz}$ at $V=5 \mathrm{~V}_{\mathrm{rms}}$ and approaching a saturated value of $f_{0} \sim 24.5 \mathrm{kHz}$ at voltages beyond $12 \mathrm{~V}_{\text {rms. }}$. This establishes that both the amplitude (Fig. 4) and frequency (Fig. 5(a)) of the voltage have a profound influence on $\lambda_{c}$ of the reflection band. The higher the amplitude (frequency) of the voltage applied across the cell gap, the lower the frequency (amplitude) required for desired $\lambda_{c}$
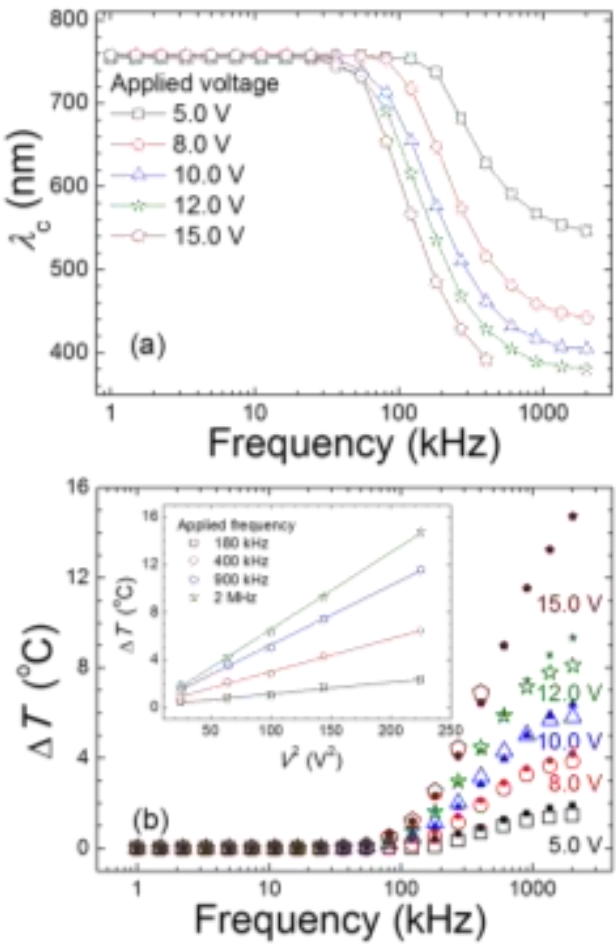

FIG. 5. Frequency-dependent (a) center wavelength and (b) temperature elevation at various $\mathrm{AC}$ voltages. The open and solid symbols in (b) represent $\Delta T$ deduced from fitting of the experimental data using Eq. (2) and measured with an IR camera, respectively.

tuning (see Eq. (6)). For instance, to achieve tunable $\lambda_{\mathrm{c}}$ from $760 \mathrm{~nm}$ to $380 \mathrm{~nm}$, one can vary the frequency from $24.5 \mathrm{kHz}$ to $2 \mathrm{MHz}$ at $V=12 \mathrm{~V}_{\mathrm{rms}}$ or from $24.5 \mathrm{kHz}$ to $403.7 \mathrm{kHz}$ at $V$ $=15 \mathrm{~V}_{\text {rms. }}$. Furthermore, Fig. 5(b) presents the frequency dependence of $\Delta T$ of the cell applied with various voltages. The $\Delta T$ data, obtained by fitting the results of Fig. 5(a) into Eq. (2), nicely match the (solid-symbol) curves acquired by IR thermography, corroborating the change in $\lambda_{\mathrm{c}}$ by voltageinduced temperature alteration. Notice that the $\Delta T(f)$ curves at various voltages can be plotted by fitting the data using Eq. (6) as well (not shown in the figure). In addition, one can see from the inset of Fig. $5(\mathrm{~b})$ that $\Delta T$ is linearly proportional to the square of $V$, conforming to the mechanism of the dielectric heating effect as described by Eq. (6). Furthermore, to verify that the abovementioned results are non-specific, we prepared another CLC cell, designated CLC-1, whose ITO resistivity (giving $R_{\mathrm{ITO}} \sim 1.6 \mathrm{k} \Omega$ ) is much larger than that of the primarily used cell $\left(R_{\mathrm{ITO}} \sim 310 \Omega\right)$ in this study, whereas other conditions, such as $A=1 \mathrm{~cm}^{2}, d=5 \mu \mathrm{m}$ and the CLC injected, for these two cells are kept identical. Figure 6 shows the dielectric spectra of CLC- 1 at $T=23{ }^{\circ} \mathrm{C}$ (Fig. 6(a)) and the frequency-dependent $\lambda_{\mathrm{c}}$ and $\Delta T$ of this cell under the application of a $15-\mathrm{V}_{\text {rms }}$ voltage (Fig. 6(b)). One can still observe from Fig. 6(a) a single Debye-like dielectric relaxation but it shifts to lower frequencies as compared with that of results shown in Fig. 2(a). By fitting the realpart dielectric spectrum into Eq. (3), the results indicate an identical value of $\mathcal{E}_{\mathrm{s}}=6.4$ and a decrease in $\mathrm{f}_{\mathrm{PR}}$ (from 440 $\mathrm{kHz}$ to $93 \mathrm{kHz}$ ) upon increasing the value of $R_{\text {Iто }}$ (from 310 $\Omega$ to $1.8 \mathrm{k} \Omega$ ). This confirms again that the unique dielectric relaxation in the complex dielectric spectra of our used CLC 

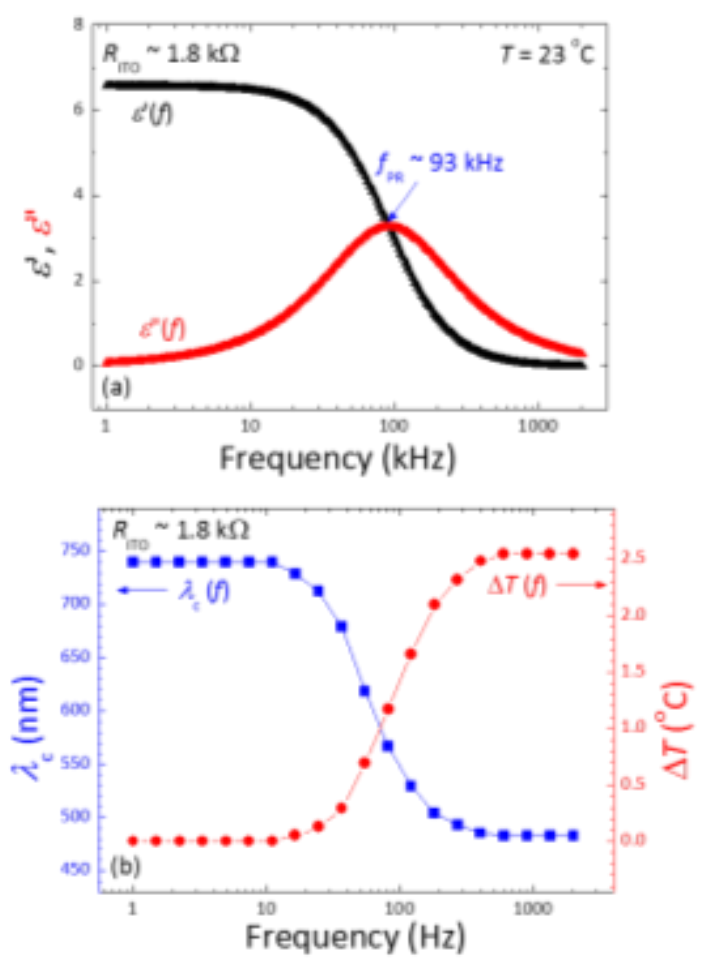

FIG. 6. (a) Complex dielectric spectra of the CLC cell with $R_{\mathrm{ITO}}=1.6 \mathrm{kHz}$ at $T=23{ }^{\circ} \mathrm{C}$ and (b) frequency-dependent $\lambda_{\mathrm{c}}$ and $\Delta T$ of this cell under a $15-\mathrm{V}$ applied voltage.

cell is attributable to the significant $R_{\mathrm{ITO}}-C_{\text {cell }}$ circuit of the cell rather than the rotation of LC molecules. Moreover, electrically tunable $\lambda_{\mathrm{c}}$ of CLC- 1 can also be realized but its tunable range (between $740 \mathrm{~nm}$ and $480 \mathrm{~nm}$ ) by $15-\mathrm{V}_{\mathrm{rms}}$ voltage in the frequency range between $1 \mathrm{kHz}$ and $2 \mathrm{MHz}$ is narrower than that of the CLC with $R_{\text {ITO }} \sim 310 \Omega$ (between $760 \mathrm{~nm}$ to $380 \mathrm{~nm}$ ) even by $V=12 \mathrm{~V}_{\mathrm{rms}}$ in the same frequency range, as shown in Fig. 6(b). Similarly, we obtain $\Delta T$ by the variation in $\lambda_{\mathrm{c}}$ according to Eq. (2). As displayed in Fig. 6(b), the frequency-dependent $\Delta T$ curve of CLC- 1 can perfectly be fitted by Eq. (6) with $f_{R}=91.2 \mathrm{kHz}$ which still follows the relation of $f_{\mathrm{R}} \sim f_{\mathrm{PR}}=93 \mathrm{kHz}$. The frequency-dependent $\Delta T$ also reflects a weakened efficacy of dielectric heating of CLC- 1 by lowered $f_{R}$; this is again in consistence with Eq. (6). Additionally, experiments using cells with various cell parameters have also been performed in our laboratory. It can be summarized based on the data that the cell gap, the electrode area, and the intrinsic dielectric anisotropy of LC materials can be used to optimize the pseudo-dielectric relaxation and the strength of dielectric heating, following Eqs. (1) and (5), respectively.

\section{CONCLUSIONS}

In summary, we have demonstrated a feasible approach enabling the electrically tunable reflective color from a CLC over the full visible spectrum. According to the thermally sensitive bandgap properties of the CLC and the cell parameters, the working principle of this approach is explicated in terms of the tunable $\lambda_{\mathrm{c}}$ of the Bragg reflection band via the voltage-induced dielectric heating effect instead of the Joule heat. Notably, the dielectric heating is predominated by the pseudo-dielectric relaxation originating from the use of the ITO cell with finite conductance. This heating effect is unique and demonstrated for its first time in this study, which should be carefully distinguished from the well-known dielectric heating effect in dual-frequency LCs explained by the dipole rotation of the molecules. Upon the application of $\mathrm{AC}$ voltage to the cell, our results validate that the tunable color (380 $\mathrm{nm} \leq \lambda_{\mathrm{c}} \leq 780 \mathrm{~nm}$ ) can be readily realized by varying either the voltage amplitude (say, $0 \mathrm{~V}_{\mathrm{rms}} \leq V \leq 12 \mathrm{~V}_{\mathrm{rms}}$ at $f=$ $2 \mathrm{MHz}$ ) or frequency (e.g., $24.5 \mathrm{kHz} \leq f \leq 2 \mathrm{MHz}$ at $V=12$ $\mathrm{V}_{\text {rms }}$ ). Because the CLC exhibits electro-thermally tunable $\lambda_{\mathrm{c}}$ between $340 \mathrm{~nm}$ at $0 \mathrm{~V}_{\mathrm{rms}}$ and $1392 \mathrm{~nm}$ at $15 \mathrm{~V}_{\mathrm{rms}}$ with a frequency of $2 \mathrm{MHz}$, true black color can be facilely obtained. The fabrication of the proposed negative CLC cell is of ease by virtue of all materials used, including the empty sandwich-type cells with ITO substrates, to be commercially available. In contrast to other electrical tuning methods, this study provides a new pathway to a wider tunable reflective color range with lower operating voltage, promoting the applicability of CLC for the design of superior color-reflective displays and electrically tunable photonic devices.

\section{ACKNOWLEDGMENTS}

This study was financially supported by the Ministry of Science and Technology, Taiwan, through Grant Nos. 1042112-M-009-008-MY3 and 106-2923-M-009-002-MY3.

\section{REFERENCES}

1. D.-K. Yang, J. L. West, L.-C. Chien, and J. W. Doane, "Control of reflectivity and bistability in displays using cholesteric liquid crystals," J. Appl. Phys. 76, 1331-1333 (1994).

2. N. Y. Ha, Y. Ohtsuka, S. M. Jeong, S. Nishimura, G. Suzaki, Y. Takanishi, K. Ishikawa, and H. Takezoe, "Fabrication of a simultaneous red-green-blue reflector using single-pitched cholesteric liquid crystals," Nat. Mater. 7, 43-47 (2008).

3. Y. Matsuhisa, Y. Huang, Y. Zhou, S.-T. Wu, R. Ozaki, Y. Takao, A. Fujii, and M. Ozaki, "Low-threshold and high efficiency lasing upon band-edge excitation in a cholesteric liquid crystal," Appl. Phys. Lett. 90, 091114 (2007).

4. C.-K. Chang, S.-W. Chiu, H.-L. Kuo, and K.-T. Tang, "Cholesteric liquid crystal-carbon nanotube hybrid architectures for gas detection," Appl. Phys. Lett. 100, 043501 (2012).

5. G. Chilaya, Cholesteric Liquid Crystals: Optics, Electro-optics, and Photo-optics, in Chirality in Liquid Crystals, edited by H.-S. Kitzerow and C. Bahr (Springer, New York, 2001), Ch. 6, pp. 159-185.

6. F. Zhang and D.-K. Yang, "Temperature dependence of pitch and twist elastic constant in a cholesteric to smectic A phase transition," Liq. Cryst. 29, 1497-1501 (2002).

7. Z. Cheng, K. Li, R. Guo, F. Wang, X. Wu, L. Zhang, J. Xiao, H. Cao, Z. Yang, and H. Yang, "Bandwidthcontrollable reflective polarisers based on the temperature-dependent chiral conflict in binary chiral mixtures," Liq. Cryst. 38, 233-239 (2011). 
8. Y. Huang, Y. Zhou, C. Doyle, and S.-T. Wu, "Tuning the photonic band gap in cholesteric liquid crystals by temperature-dependent dopant solubility," Opt. Express 3, 1236-1242 (2006).

9. T. J. White, M. E. McConney, and T. J. Bunning, "Dynamic color in stimuli-responsive cholesteric liquid crystals," J. Mater. Chem. 20, 9832-9847 (2010).

10. T.-H. Lin, C.-H. Chen, Y. Chen, T. Wei, C.-W. Chen, and A. Y.-G. Fuh, "Electrically controllable laser based on cholesteric liquid crystal with negative dielectric anisotropy," Appl. Phys. Lett. 88, 061122 (2006).

11. C. A. Bailey, V. P. Tondiglia, L. V. Natarajan, M. M. Duning, R. L. Bricker, R. L. Sutherland, T. J. White, M. F. Durstock, and T. J. Bunning, "Electromechanical tuning of cholesteric liquid crystals,” J. Appl. Phys. 107, 013105 (2010).

12. L. V. Natarajan, J. M. Wofford, V. P. Tondiglia, R. L. Sutherland, H. Koerner, R. A. Vaia, and T. J. Bunning, "Electro-thermal tuning in a negative dielectric cholesteric liquid crystal material," J. Appl. Phys. 103, 093107 (2008).

13. S. S. Choi, S. M. Morris, W. T. S. Huck, and H. J. Coles, Wavelength tuning the photonic band gap in chiral nematic liquid crystals using electrically commanded surfaces,"” Appl. Phys. Lett. 91, 231110 (2007).

14. S. S. Choi, S. M. Morris, W. T. S. Huck, and H. J. Coles, "Electrically tuneable liquid crystal photonic bandgaps,"Adv. Mater. 21, 3915-3918 (2009).

15. J. Xiang, Y. Li, Q. Li, D. A. Paterson, J. M. D. Store, C. T. Imrie, and O. D. Lavrentovich, "Electrically tunable selective reflection of light from ultraviolet to visible and infrared by heliconical cholesterics," Adv. Mater. 27, 3014-3018 (2015).

16. M. Schadt, "Dielectric heating and relaxations in nematic liquid crystals," Mol. Cryst. Liq. Cryst. 66, 319-336 (1981).

17. C.-H. Wen and S.-T. Wu, "Dielectric heating effects of dual-frequency liquid crystals," Appl. Phys. Lett. 86, 231104 (2005).

18. Y. Yin, S. V. Shiyanovskii, and O. D. Lavrentovich, "Electric heating effects in nematic liquid crystals," J Appl. Phys. 100, 024906 (2006).

19. P. Perkowski, D. Lada, K. Ogrodnik, J. Rutkowska, W. Piecek, and Z. Raszewski, "Technical aspects of dielectric spectroscopy measurements of liquid crystals," Opto-Electron. Rev. 16, 271-276 (2008).

20. S.-Y. T. Tzeng, C.-N. Chen, and Y. Tzeng, "Thermal tuning band gap in cholesteric liquid crystals," Liq. Cryst. 37, 1221-1224 (2010).

21. P. N. Keating, "A theory of the cholesteric mesophase" Mol. Cryst. Liq. Cryst. 8, 315-326 (1969).

22. Y.-C. Hsiao, Z.-H. Yang, D. Shen, and W. Lee, "Technical aspects of dielectric spectroscopy measurements of liquid crystals," Adv. Opt. Mater. 6, 1701128 (2018).

23. P. Perkowski, "How to determine parameters of soft mode from dielectric spectroscopy performed using cells with ITO electrodes?" Opto-Electron. Rev. 19, 76-82 (2011). 